\title{
Innate immune stimulation of whole blood reveals IFN-1 hyper-responsiveness in type 1 diabetes
}

\author{
Kameron B. Rodrigues ${ }^{1,2}$ (D) Matthew J. Dufort $^{3}$ (D) Alba Llibre $^{4} \cdot$ Cate Speake $^{5} \cdot$ M. Jubayer Rahman ${ }^{1}$. \\ Vincent Bondet ${ }^{4} \cdot$ Juan Quiel ${ }^{1} \cdot$ Peter S. Linsley $^{3} \cdot$ Carla J. Greenbaum $^{5} \cdot$ Darragh Duffy $^{4} \cdot$ Kristin V. Tarbell $^{1,6} \mathbb{C}$
}

Received: 16 December 2019 / Accepted: 17 March 2020 / Published online: 5 June 2020

(C) This is a U.S. government work and not under copyright protection in the U.S.; foreign copyright protection may apply 2020

\begin{abstract}
Aims/hypothesis Self-antigen-specific $\mathrm{T}$ cell responses drive type 1 diabetes pathogenesis, but alterations in innate immune responses are also critical and not as well understood. Innate immunity in human type 1 diabetes has primarily been assessed via gene-expression analysis of unstimulated peripheral blood mononuclear cells, without the immune activation that could amplify disease-associated signals. Increased responsiveness in each of the two main innate immune pathways, driven by either type 1 IFN (IFN-1) or IL-1, have been detected in type 1 diabetes, but the dominant innate pathway is still unclear. This study aimed to determine the key innate pathway in type 1 diabetes and assess the whole blood immune stimulation assay as a tool to investigate this.

Methods The TruCulture whole blood ex vivo stimulation assay, paired with gene expression and cytokine measurements, was used to characterise changes in the stimulated innate immune response in type 1 diabetes. We applied specific cytokine-induced signatures to our data, pre-defined from the same assays measured in a separate cohort of healthy individuals. In addition, NOD mice were stimulated with $\mathrm{CpG}$ and monocyte gene expression was measured.

Results Monocytes from NOD mice showed lower baseline vs diabetes-resistant B6.g7 mice, but higher induced IFN-1associated gene expression. In human participants, ex vivo whole blood stimulation revealed higher induced IFN-1 responses in type 1 diabetes, as compared with healthy control participants. In contrast, neither the IL-1-induced gene signature nor response to the adaptive immune stimulant Staphylococcal enterotoxin B were significantly altered in type 1 diabetes samples vs healthy control participants. Targeted gene-expression analysis showed that this enhanced IFN response was specific to IFN-1, as IFN- $\gamma$-driven responses were not significantly different.

Conclusions/interpretation Our study identifies increased responsiveness to IFN-1 as a feature of both the NOD mouse model of autoimmune diabetes and human established type 1 diabetes. A stimulated IFN-1 gene signature may be a potential biomarker for type 1 diabetes and used to evaluate the effects of therapies targeting this pathway.
\end{abstract}

Kameron B. Rodrigues and Matthew J. Dufort contributed equally to this study.

Electronic supplementary material The online version of this article (https://doi.org/10.1007/s00125-020-05179-4) contains peer-reviewed but unedited supplementary material, which is available to authorised users.

Darragh Duffy

darragh.duffy@pasteur.fr

Kristin V. Tarbell

ktarbell@amgen.com

1 Immune Tolerance Section, Diabetes Branch, National Institute of Diabetes and Digestive and Kidney Diseases, Bethesda, MD, USA

2 Present address: Pathology Department, Stanford University School of Medicine, Palo Alto, CA, USA
3 Systems Immunology Division, Benaroya Research Institute at Virginia Mason, Seattle, WA, USA

4 Immunobiology of Dendritic Cells/Inserm U1223, Département d'Immunologie, Institut Pasteur, 25 rue de Dr. Roux, 75724 Paris, France

5 Diabetes Program, Benaroya Research Institute at Virginia Mason, Seattle, WA, USA

6 Present address: Amgen Discovery Research, 1120 Veterans Blvd, South San Francisco, CA 94080, USA 


\section{Research in context}

\section{What is already known about this subject?}

- Innate immune responses contribute to the pathogenesis of many autoimmune diseases, including type 1 diabetes, and genetic risk alleles associated with type 1 diabetes encode genes expressed in innate immune cells

- Previous work has suggested that both IL-1- and IFN-driven response may have a role in type 1 diabetes

\section{What is the key question?}

- Can ex vivo whole blood immune stimulation facilitate identification of important innate immune alterations in individuals with type 1 diabetes?

\section{What are the new findings?}

- Immune cells from individuals with type 1 diabetes and NOD mice display lower expression of IFN-1-driven genes without stimulation, but higher IFN-1 responses with stimulation

- No significant differences were observed between individuals with type 1 diabetes and healthy control participants in either IL-1 or IFN- $\gamma$ driven gene signatures, or Staphylococcal enterotoxin B-induced gene expression

\section{How might this impact on clinical practice in the foreseeable future?}

- Stimulated IFN-1-driven gene expression may allow characterisation of heterogeneity in innate immune responses within individuals with type 1 diabetes that could be used as a biomarker to identify the subset of individuals with type 1 diabetes that may respond to therapies targeting the IFN-1 pathway

Data availability Mouse gene expression data are found in the gene expression omnibus (GEO) repository, accession GSE146452 (www.ncbi.nlm.nih.gov/geo/query/acc.cgi?acc=GSE146452). Nanostring count data from the human experiments were deposited in the GEO repository, accession GSE146338 (www.ncbi.nlm.nih.gov/geo/query/acc.cgi?acc= GSE146338). Data files and R code for all analyses are available at https://github.com/rodriguesk/T1D_truculture_diabetologia.

Keywords Cytokines · Diabetes mellitus, type $1 \cdot$ Enterotoxins $\cdot$ Immunity, innate $\cdot$ Interleukin- $1 \cdot$ Leukocytes, mononuclear Monocytes $\cdot$ Non-obese diabetic mice (mice, inbred NOD) · Type 1 IFN

\begin{tabular}{ll}
\multicolumn{2}{l}{ Abbreviations } \\
BRI & Benaroya Research Institute \\
cDC & Conventional dendritic cell \\
CFB & Complement factor B \\
IFNAR & IFN- $\alpha$ receptor \\
ISG & IFN-stimulated gene \\
LoD & Limit of detection \\
LPS & Lipopolysaccharide \\
MSigDB & Molecular Signatures Database \\
PBMC & Peripheral blood mononuclear cells \\
PolyIC & Polyinosinic-polycytidylic acid \\
SEB & Staphylococcal enterotoxin B \\
Th1 & T helper 1
\end{tabular}

\section{Introduction}

Type 1 diabetes is an autoimmune disease that manifests in adaptive immune responses targeting the insulin-producing beta cells of the endocrine pancreas. However, alterations in innate immune responses also drive autoimmune pathogenesis, with involvement in both the initial break in tolerance and the later failure of regulation [1-4]. Many known type 1 diabetes genetic risk alleles encode genes expressed by antigen presenting cells or other innate immune cells [5]. Understanding how innate immune responses are altered in type 1 diabetes and other autoimmune diseases is important for elucidating pathogenic pathways that could be targeted with new treatments. Within the spectrum of innate immune responses, inflammatory responses can generally be categorised into type 1 IFN (IFN-1)- or IL-1 dominant [6-10]. IL-1 and IFN-1 responses can counter-regulate each other and are needed for responses to different types of pathogens: IFN-1 increases antigen presentation and is primarily involved in viral responsiveness, whereas IL-1 stimulates secretion of inflammatory cytokine, such as IL-6, and drives responses to bacterial infection $[6,11]$. Both types of responses have been postulated to play a role in the pathogenesis of type 1 diabetes and other autoimmune diseases [12, $13]$. 
Autoimmune diabetes-susceptible NOD mice display alterations in both IFN-1 and IL-1 responses. Higher IFN-1 responses are observed in young (2-4-week-old) NOD mice and blocking the IFN- $\alpha$ receptor (IFNAR) at this age inhibits diabetes $[14,15]$, suggesting that higher IFN-1 responsiveness may contributes to the initial loss of self-tolerance. However, we have shown that myeloid cells from older prediabetic NOD mice display impaired expression of IFN-1-induced genes but higher IL-1-associated gene expression [16].

Previous studies have not provided a clear picture of how innate immune responses are altered in human type 1 diabetes. Viral infections, and the associated IFN response, are a potential environmental trigger for type 1 diabetes in genetically susceptible individuals [17], and individuals at risk for developing type 1 diabetes have shown higher expression of IFN-1 response genes (IFN-stimulated genes [ISGs]) in peripheral blood mononuclear cells (PBMCs) without ex vivo stimulation. This IFN signature has been shown to correlate with the development of islet autoantibodies, but wanes before the development of overt disease [18, 19]. Furthermore, pancreatic islets from individuals with type 1 diabetes may display IFN hyper-responsiveness [20-22]. Increased IL-1 has also been associated with type 1 diabetes [23]. For example, monocytes from individuals with type 1 diabetes can make more IL1 and IL-6 without stimulation [24]. Increased IL-1-associated responses have been observed at the time of diagnosis; these increases were extinguished with IL-1 receptor antagonist treatment $[25,26]$. Although blocking IL-1 signals leads to clinical improvement in other autoimmune diseases, such as rheumatoid arthritis, and in type 2 diabetes [27, 28], two type 1 diabetes trials that blocking IL-1 signals with either anakinra or canakinumab failed to alter insulin secretion in individuals with new-onset type 1 diabetes [29]. Together, these studies show that, in different contexts, type 1 diabetes has been associated with innate immune responses dominated by both IFN1 and IL-1. Less is known about innate immunity in individuals with established type 1 diabetes.

One potential limitation of many previous studies on innate immune changes associated with human autoimmunity is the lack of relevant innate stimulation that can increase the response range. Although some prior studies have assessed cytokine responses after innate stimulation of either PBMCs or purified cell populations, a mix of either increased IL-1- or IFN-1-associated responses were associated with type 1 diabetes [23, 30]. However, the process of cellular isolation can alter phenotypes and may eliminate the inter-cell crosstalk that occurs in vivo. In contrast, a whole blood assay (TruCulture) was used by the Milieu Intérieur project to standardise human immune stimulation directly, ex vivo; this has facilitated measurements of individual response variation, and categorisation of stimulus-specific immune responses [31, 32]. This assay has lower technical variation than PBMCbased assays and includes innate immune cells not present in
PBMCs [33]. In this study, we compared monocyte gene expression after $\mathrm{CpG}$ stimulation in NOD and control mice, and measured gene expression and cytokine responses after ex vivo innate immune stimulation in 17 individuals with established type 1 diabetes and 17 age-matched control participants. A better understanding of innate immune responses in human type 1 diabetes and related mouse models could help identify the innate pathways that could be targeted therapeutically for successful disease modification.

\section{Methods}

\section{Mouse CpG stimulation and monocyte gene expression analysis}

Female 8-10-week-old NOD and control B6.g7 mice (Jackson Laboratories, Ellsworth, ME, USA), 3-4 mice/group, were injected intravenously with $170 \mu \mathrm{PBS}+$ $30 \mu$ 1,2-Dioleoyloxy-3-trimethylammonium propane (DOTAP; Roche, Indianapolis, IN, USA), with and without 5 g CpG-A (2216; Invivogen, San Diego, CA, USA). After $6 \mathrm{~h}$, spleen cells were harvested and $\mathrm{Ly}_{6 \mathrm{C}}{ }^{+}$monocytes were sorted. Extracted RNA was sequenced and analysed, as previously described [16, 34, 35]. Details and other analysis of the unstimulated mouse samples used in this study were previously published [16]. The experiments were not replicated, with each mouse acting as a biological replicate.

\section{Human Study design}

Samples from 17 individuals with type 1 diabetes and 17 healthy control participants matched for age \pm 1 year were collected, concurrently, over 6 months, usually as agematched pairs that were collected within 1 week of each other (Table 1 and electronic supplementary material [ESM] Table 1). Individuals with type 1 diabetes had history of clinical diagnosis and presence of type 1 diabetes-associated autoantibodies, were diagnosed 3 months to 8 years prior to blood sample collection, and had good glucose control, as represented by $\mathrm{HbA}_{1 \mathrm{c}}<63.9 \mathrm{mmol} / \mathrm{mol}(8 \%)$, although $\mathrm{HbA}_{1 \mathrm{c}}$ at time of collection was not an exclusion criterion. Healthy control participants had no first-degree relatives with type 1 diabetes. Groups were not sex-matched but gene expression changes were not associated with sex (ESM Fig. 1). All participants were enrolled in the Benaroya Research Institute (BRI) Immune Mediated Disease Registry. All participants fasted overnight prior to the blood draw. $\mathrm{HbA}_{1 \mathrm{c}}$ was measured under Clinical Laboratory Improvement Amendments (CLIA)approved protocols at the University of Washington's Northwest Lipid Metabolism and Diabetes Research Laboratories. Each sample was only measured once; repeat measurements were not taken from the same sample. 
Table 1 Characteristics of human participants

\begin{tabular}{|c|c|c|c|c|c|c|}
\hline Group & Sex (male/female) & Age (years) & Disease duration (years) & $\mathrm{HbA}_{1 \mathrm{c}}(\mathrm{mmol} / \mathrm{mol})$ & $\mathrm{HbA}_{1 \mathrm{c}}(\%)$ & Glucose (mmol/l) \\
\hline Control & $4 / 13$ & $26.7 \pm 2.8$ & - & - & - & - \\
\hline Type 1 diabetes & $8 / 9$ & $26.7 \pm 2.8$ & $4.3 \pm 2.0$ & $51.2 \pm 13.7$ & $6.8 \pm 1.3$ & $7.71 \pm 2.26$ \\
\hline
\end{tabular}

Data are presented as mean $\pm \mathrm{SD}$ unless otherwise indicated

\section{TruCulture tube collection and quantification of cytokine and gene expression}

Peripheral blood was drawn directly into TruCulture tubes (Myriad RBM, Austin, TX, USA) for stimulation following manufacturer's instructions, and as previously described [32]. Tubes were pre-loaded with one of the following immune stimuli: Staphylococcal enterotoxin B (SEB; $0.4 \mu \mathrm{g} / \mathrm{ml}$ ), IL$1 \beta(25 \mathrm{ng} / \mathrm{ml}$ or $1760 \mathrm{IU} / \mathrm{ml})$, lipopolysaccharide (LPS; $1 \mathrm{ng} / \mathrm{ml})$, IFN- $\beta$ (1000 IU/ml), polyinosinic-polycytidylic acid (polyIC; $20 \mu \mathrm{g} / \mathrm{ml}$ ), with stimulus concentrations previously determined [31], or a no-stimulus null control. After blood draw and incubation, the supernatant and cells were separated for downstream assays of secreted proteins and mRNA, respectively.

Cytokines were quantified in TruCulture tube supernatants using the OptiMAP Luminex panel (Myriad RBM), following manufacturer protocols. IFN- $\alpha$ and IFN- $\beta$ were quantified using Simoa assays (Quanterix, Billerica, MA, USA), as described previously $[36,37]$. The limit of detection (LoD) was $0.6 \mathrm{fg} / \mathrm{ml}$ for IFN- $\alpha$ and $0.45 \mathrm{pg} / \mathrm{ml}$ for IFN- $\beta$.

Gene expression was quantified in stimulated whole blood samples using the nCounter Human Immunology kit v2 (NanoString, Seattle, WA, USA). Total mRNA was extracted from TruCulture cell pellets stabilised in Trizol LS (SigmaAldrich, St Louis, MO, USA), using the NucleoSpin 96 RNA tissue kit protocol (Macherey-Nagel, Düren, Germany), as previously described [32]. For each sample, $100 \mathrm{ng}$ of total RNA was analysed.

\section{Gene expression analysis}

Data processing and normalisation Normalisation of Nanostring nCounter data was performed as described previously [32], using nSolver (NanoString; v. 2.5) for analysis of quality and negative and positive controls. Differences in RNA input were normalised using six housekeeping genes (EEF1G, RPL19, PPIA, TBP, G6PD, POLR2A), selected using the geNorm method [38]. We estimated a lower LoD of 4 counts [39], and retained 520 genes with expression above this $L o D$ in $\geq 1 / 3$ of samples from any stimulation condition in both participant groups. Multi-dimensional scaling (MDS) of gene counts revealed batch differences associated with the sample quantification method; ComBat [40] in the sva R package [41] was used to remove this batch effect prior to downstream analyses.

Differential expression analysis Differential gene expression was determined using limma-voom [42], accounting for nonindependence of samples from the same participant using limma's duplicateCorrelation. Between-group comparisons of the stimulation effect used the interaction term between disease status and stimulus. Multiple comparisons were adjusted using the Benjamini-Hochberg false discovery rate (FDR). Heatmaps were generated using ComplexHeatmap [43].

Gene set and signature score analyses We determined differential expression of pre-defined gene sets using ROAST [44]. For human samples, we used gene sets from two sources; first, we used hallmark IFN response gene sets from the Molecular Signatures Database (MSigDB) [45]. For more refined stimulus-specific responses, we used genes that have increased expression after TruCulture tube stimulation with IFN- $\alpha$, IFN- $\beta$, IFN- $\gamma$ or IL-1 $\beta$ [32] and have an ortholog to mouse ISGs. For mouse samples, we used genes that respond to IFN- $\alpha$ stimulation in vivo in many different cell types [10] and genes specifically induced by IFN- $\beta$ [32]. ESM Table 2 shows details of gene sets. Signature scores for each gene set are the sample-level means of the gene-level z scores of expression or log-fold change.

\section{Statistics, visualisations and code}

Samples were not randomised and the experimenters were not blinded. All statistical analyses were performed using R v. 3.5.2 [46], with visualisations using $\mathrm{R}$ base graphics and ggplot2 [47]. $t$ tests were two sided and data are expressed as means (SEM), unless otherwise indicated. Specific tests and significance thresholds are listed in the text; where not noted, tests were two-tailed and $p<0.05$ was considered significant.

\section{Study approval}

Animals were housed in specific pathogen-free conditions and handled according to guidelines provided by the Animal Care and Use Committee of the National Institute of Diabetes 
and Digestive and Kidney Diseases (NIDDK) at the National Institutes of Health (NIH). All human samples were prospectively obtained under written informed consent. All study protocols were approved by the BRI Institutional Review Board (IRB).

\section{Results}

\section{Higher CpG-stimulated IFN-1 responses in monocytes from diabetes-susceptible NOD mice}

To determine if the acute IFN-1 response is altered in NOD mice, we analysed gene expression in monocytes from NOD and B6.g7 mice $6 \mathrm{~h}$ after in vivo stimulation with $\mathrm{CpG}$. Gene set analysis showed that the expression of IFN-1-induced genes was lower in unstimulated monocytes from NOD vs diabetes-resistant B6.g7 mice, as we have shown previously [16], but these same IFN-1-stimulated genes were more strongly upregulated in NOD monocytes after stimulation with $\mathrm{CpG}$ (Fig. 1a, b). A more restricted set of IFN-response genes that are orthologs to human IFN-response genes also showed this pattern of lower unstimulated but higher stimulated expression in NOD mice (Fig. 1c). Therefore, monocytes from NOD mice with chronic autoimmunity display lower baseline levels and higher induction of IFN-1 response genes with $\mathrm{CpG}$ stimulation, as compared with those from control B6.g7 mice.

\section{Innate immune stimuli induce changes in gene expression and upregulate inflammatory cytokine levels in human whole blood samples}

TruCulture ex vivo whole blood stimulation system was used to measure immune response differences between individuals with type 1 diabetes and healthy control participants. Five stimuli were chosen: SEB to induce T cell responses; an IFN-1 response induced directly via IFNAR with IFN- $\beta$; an IFN-1 response induced indirectly via pattern recognition receptors, Toll-like receptor 3 (TLR3) and retinoic acidinducible gene I (RIG-I), using polyIC; LPS to induce both type 1 and type 2 IFN and IL- 1 responses; IL-1 $\beta$ [31]. Blood was incubated with the indicated stimuli, and cell pellets and supernatants collected to measure gene expression and cytokine protein levels, respectively, as previously described (Fig. 2a) $[31,32]$. As expected, these innate stimuli induced cytokine expression in samples from both type 1 diabetic and healthy control participants, but no significant differences were observed between the type 1 diabetes and healthy control group in the analytes examined, after adjusting for multiple tests (ESM Fig. 2). Five-hundred and twenty genes from the Nanostring human immunology panel with measurable expression in at least one condition were included. Gene expression induced by distinct stimuli showed stimulusspecific patterns (Fig. 2b) and clustered separately from each other by principal component analysis (PCA; ESM Fig. 3). As previously described, stimulation with IL-1 $\beta$ elicited fewer gene expression changes as compared with other stimuli, and samples stimulated with polyIC and IFN- $\beta$ elicited similar gene expression changes as compared with other stimuli, as captured by PC2 (Fig. 2 and ESM Fig. 3) [32]. Therefore, the expected upregulation of cytokine and gene expression with stimulation was observed.

\section{Lower IFN-1 response gene expression in unstimulated samples}

Without specific immune stimulation (null stimulation), gene expression in the type 1 diabetes and healthy control group were similar, with only three genes showing significant differential expression (Fig. 3a, ESM Table 3). Specific modules of gene expression that can separate responses to key cytokines, including IFN- $\gamma$, IFN- $\beta$ and IL- $1 \beta$, were previously defined by the Milieu Intérieur Consortium, who used the approach in healthy donors [32]. The genes in these modules are the ones that are most distinct from induction by other cytokine stimuli. To better understand baseline, unstimulated human ISG expression, we applied these cytokine-specific gene expression modules to the TruCulture null samples. Using this method, we found that, without specific stimulation, the IFN-1 gene score was significantly lower in type 1 diabetes (adjusted $p=0.036$ ), but this was not the case with the type 2 IFN or IL1 gene scores (adjusted $p$ values: 0.93 and 0.12 , respectively), as compared with healthy control participants without stimulation (Fig. 3b-d). Individual Milieu Intérieur-derived IFN-1 signature genes are shown in the heatmap in Fig. 3e. Although the effect size without stimulation is small, this suggests that, as observed in NOD mice (Fig. 1a, c) [16], individuals with type 1 diabetes may have reduced tonic IFN-1 signalling.

\section{SEB-driven and IFN- $\gamma$-specific gene expression does not differ in type 1 diabetes}

Adaptive immune responses, including IFN- $\gamma$-producing $\mathrm{T}$ helper 1 (Th1) cells, have been shown to be critical for type 1 diabetes pathogenesis $[48,49]$. Thus, we examined gene expression changes after exposure to the adaptive stimulus SEB and analysed IFN- $\gamma$-driven changes in gene expression. Although SEB stimulation induced robust gene expression changes (Fig. 2b), no significant differences in gene expression were observed between the type 1 diabetes and healthy control group (Fig. 4a). The Milieu Intérieur-derived IFN- $\gamma$ induced gene expression module was applied to the two most relevant stimuli, namely SEB and LPS, but no significant differences were observed between type 1 diabetes and healthy control participants in this IFN- $\gamma$ signature (Fig. 4b). 
a

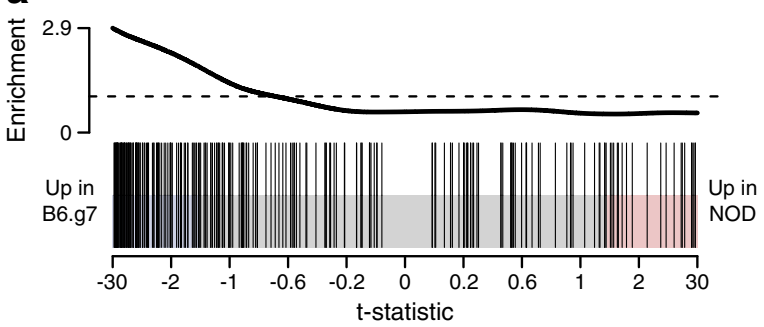

b

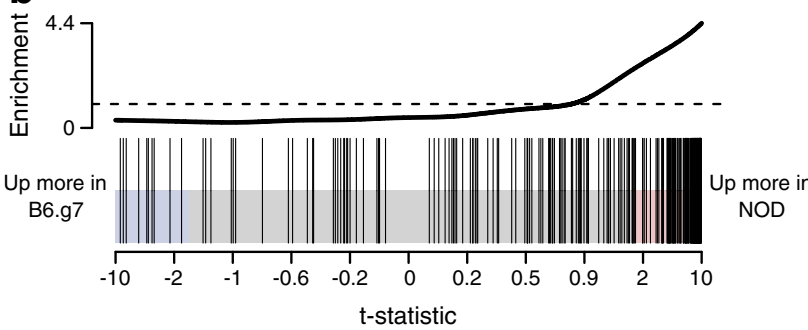

C

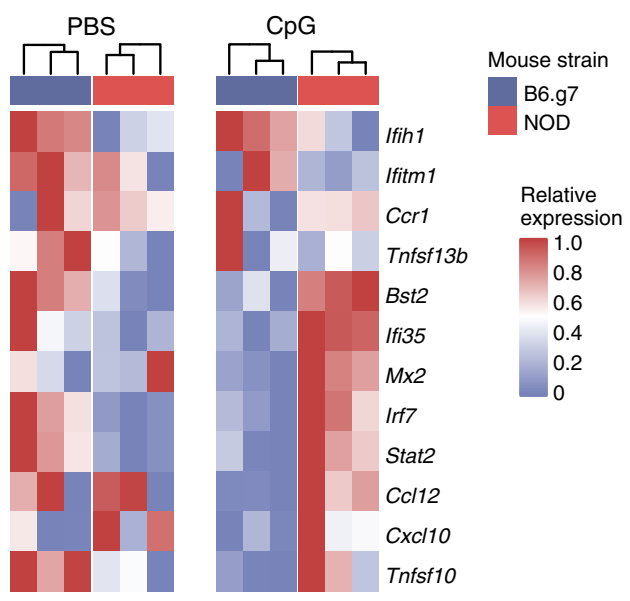

Fig. 1 Lower baseline and higher stimulated IFN-1-induced gene expression in NOD monocytes compared with control mice. NOD and control B6.g7 mice were injected with either PBS or $\mathrm{CpG}$ and, $6 \mathrm{~h}$ later, monocytes were sorted from isolated spleen cells for RNA sequencing $(n=3)$. (a, b) Gene set enrichment analysis of IFN- $\alpha$-induced genes, with decreased expression in NOD mice after null stimulation $(\mathbf{a} ; p=0.016)$ and increased expression after $\mathrm{CpG}$ stimulation $(\mathbf{b} ; p=0.0013)$. Genes

Similarly, at the protein level, IFN- $\gamma$ was not differentially induced in type 1 diabetes vs healthy control participants (Fig. 4c). Other cytokines strongly induced by SEB stimulation (IL-17, granulocyte-macrophage colony-stimulating factor [GM-CSF] and IL-2) were not differentially upregulated in type 1 diabetes as compared with healthy control participants (ESM Fig. 2). Therefore, no significant type 1 diabetes-
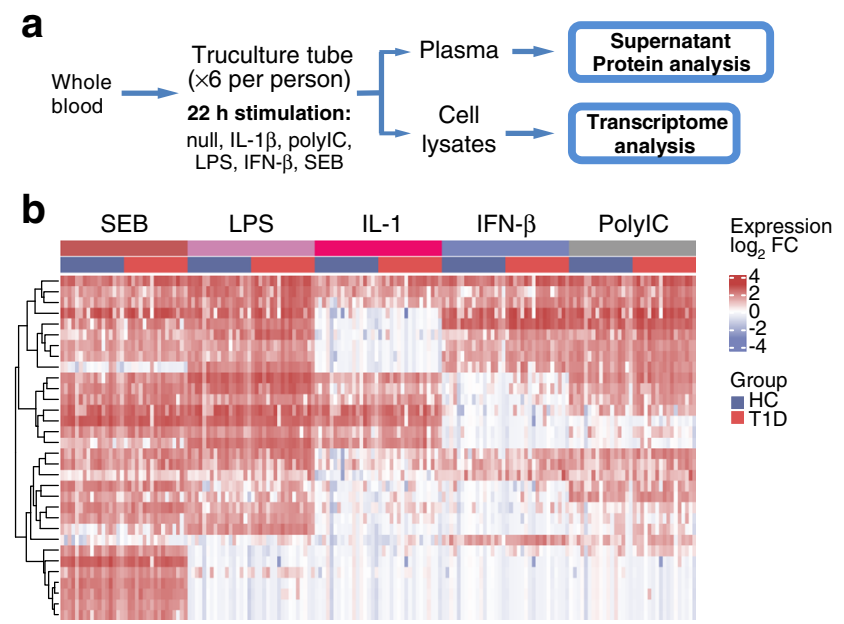

Fig. 2 Innate and adaptive stimuli induce gene expression changes in samples from both type 1 diabetes (T1D) and healthy control (HC) participants. (a) Experimental setup for collection of ex vivo-stimulated whole blood samples. (b) Heatmap of stimuli-induced genes, including all participants and all stimuli. For each stimulation, the ten most significantly upregulated genes with a fold change (FC) $\geq 2$ are shown are rank-ordered by the $t$-statistic comparing NOD with control mice; genes that are more highly expressed in NOD are on the positive side of the $t$-statistic scale. Position on the $x$-axis indicates rank. Significance values derived using limma ROAST. (c) Heatmap showing expression of genes in the Milieu Intérieur-derived IFN- $\beta$ signature in sorted monocytes from diabetes-susceptible NOD or control B6.g7 mice after either control (PBS) or CpG stimulation

associated differences were observed in the adaptive immune responses when induced by SEB or IFN- $\gamma$.

\section{IL-1 induced gene expression is not significantly altered in type 1 diabetes}

IL-1 is a key innate immune signal that may contribute to type 1 diabetes pathogenesis $[16,26,50]$. Surprisingly, IL- $1 \beta$ stimulation resulted in only two genes being differentially induced when comparing type 1 diabetes and the healthy control group (Fig. 5a), though IL-1 $\beta$ stimulation overall induced smaller changes in gene expression as compared with other stimuli (Fig. 2b) [32].

LPS also induces IL-1-specific gene expression, and type 1 diabetes-associated changes in LPS-stimulated responses were observed (LPS vs null), with ten genes being more significantly induced by LPS in the type 1 diabetes vs healthy control group, and two genes (NCF4 and S100A9) being less induced (Fig. 5b, ESM Table 3). However, LPS also can induce IFN-1 responses. For example, LPS-stimulated complement factor $\mathrm{B}(C F B)$ is higher in individuals with type 1 diabetes, but stimulation with IFN-1 has been previously shown to induce higher CFB expression than stimulation with IL-1 or IFN- $\gamma$ [51]. To further understand IL-1-driven changes in gene expression and separate them from other innate immune responses, the Milieu Intérieur-derived IL-1-specific gene module was applied to both IL-1 and LPS stimuli. LPS stimulation gave the highest level of IL-1-induced gene expression (ESM Table 3) but no significant differences in 

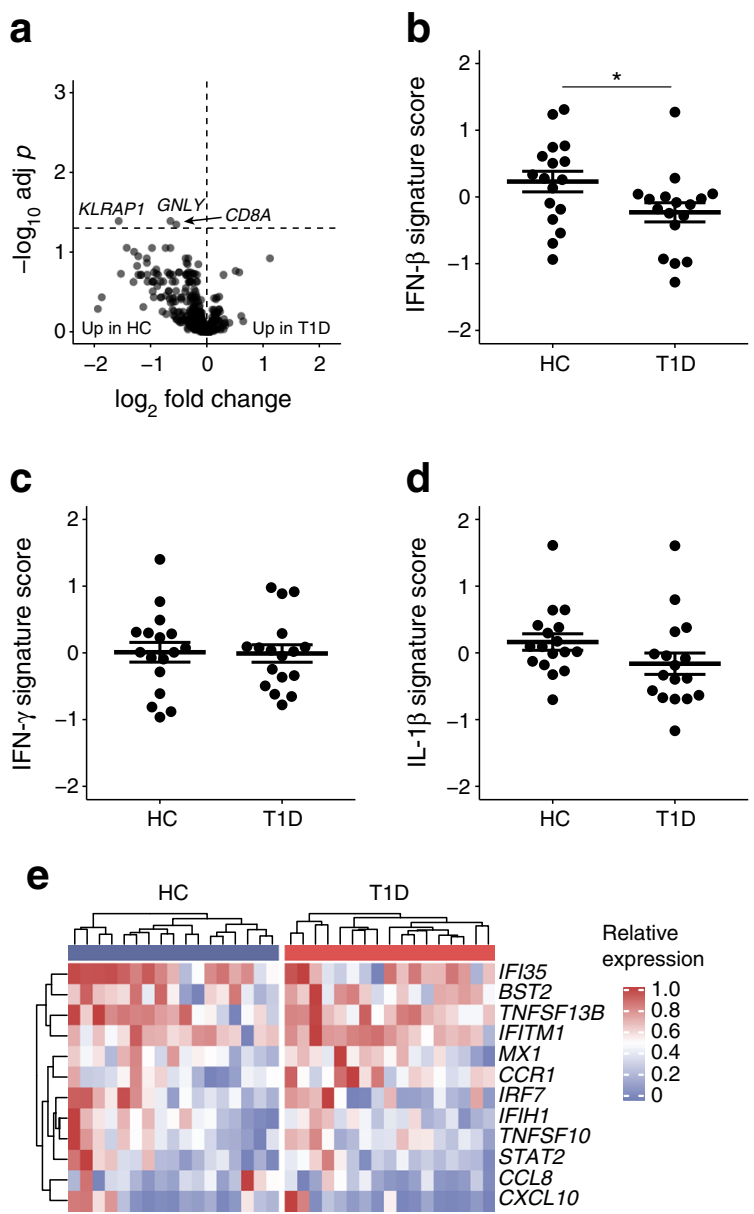

Fig. 3 Unstimulated samples show lower type 1 diabetes(T1D)-associated IFN- $\beta$-driven gene expression. (a) Volcano plot showing differential gene expression between the T1D and healthy control (HC) groups in the null stimulation tube. Horizontal dashed line indicates adjusted $(\operatorname{adj}) p=$ 0.05 ; vertical dashed line indicates log fold change equals 0 . (b-d) Gene signature scores in null-stimulated samples for Milieu Intérieur-derived IFN- $\beta$ - (b), IFN- $\gamma$ - (c) and IL- $1 \beta$ - (d) specific gene sets. For IFN- $\beta, p=$ 0.036; for IFN- $\gamma, p=0.93$; for IL-1 $\beta, p=0.12$. (e) Heatmap of Milieu Intérieur-derived IFN- $\beta$ genes in the null-stimulated samples. $* p<0.05$

the IL-1 signature were observed between the type 1 diabetes and healthy control group (Fig. 5c). Together these data show that the IL-1 response, induced either by LPS or IL-1 itself, is not significantly altered in type 1 diabetes vs healthy participants. In addition, some of the LPS-induced changes in gene expression in type 1 diabetes may be due to the effects of increased IFN-1 signals.

\section{Preferential induction of IFN-1 response genes in type 1 diabetes as compared with healthy control participants}

Next, we compared differences between individuals with type 1 diabetes and healthy control participants following stimulation with polyIC and IFN- $\beta$, both strong inducers of the IFN-1 response. We found 21 genes that were more highly
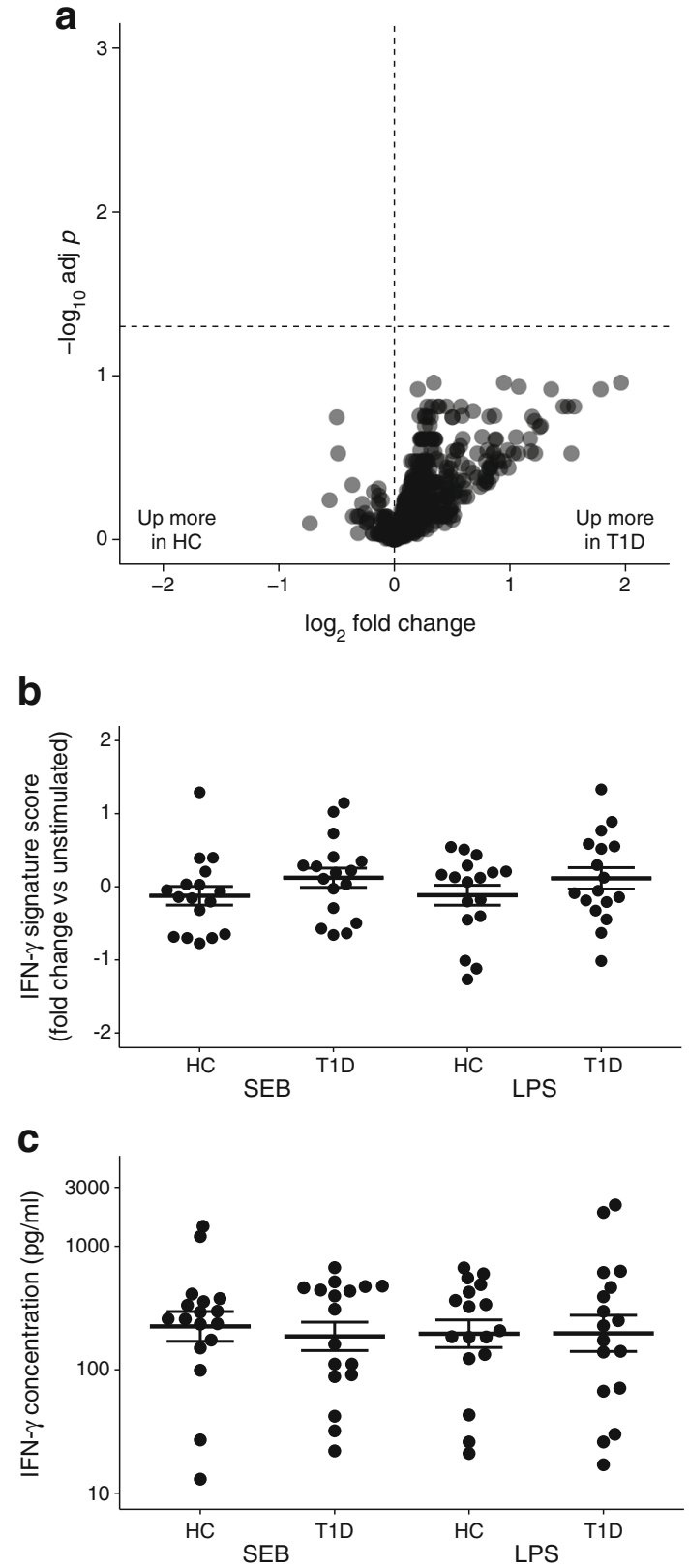

Fig. 4 SEB and IFN- $\gamma$ responses are similar in type 1 diabetes (T1D) and healthy control (HC) participants. (a) Volcano plot comparing SEBinduced gene expression in T1D vs HC. Horizontal dashed line indicates adjusted (adj) $p=0.05$; vertical dashed line indicates log fold change equals 0 . (b) Milieu Intérieur-derived IFN- $\gamma$-specific gene signature scores for samples from T1D or HC after stimulation with SEB or LPS. For SEB, $p=0.19$; for LPS, $p=0.26$. (c) IFN- $\gamma$ protein levels quantified from supernatants of T1D or HC samples stimulated with SEB or LPS. For SEB, $p=0.63$; for LPS, $p=0.99$. Values shown on $\log _{10}$ scale

upregulated in individuals with type 1 diabetes as compared with control participants after IFN- $\beta$ stimulation (Fig. 6a), and 14 genes after polyIC stimulation (Fig. 6b). No genes showed significantly higher induction in healthy control participants after either stimulus. Upon subsetting the analysis to genes in the Milieu Intérieur-derived IFN- $\beta$ module to assess IFN-1- 
a

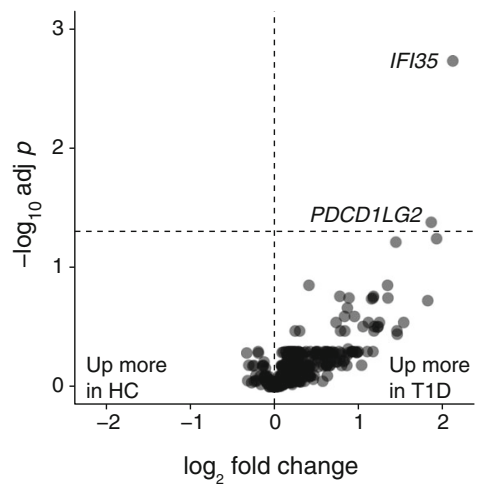

b

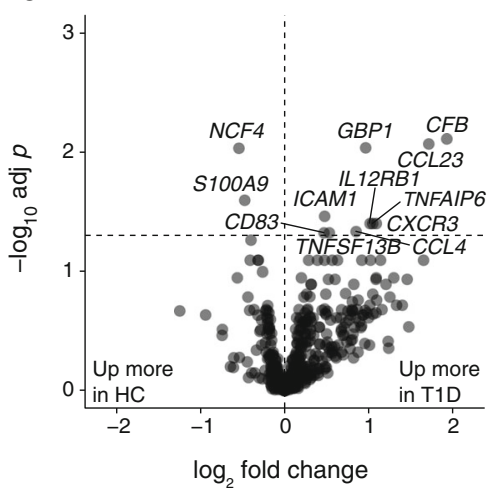

C

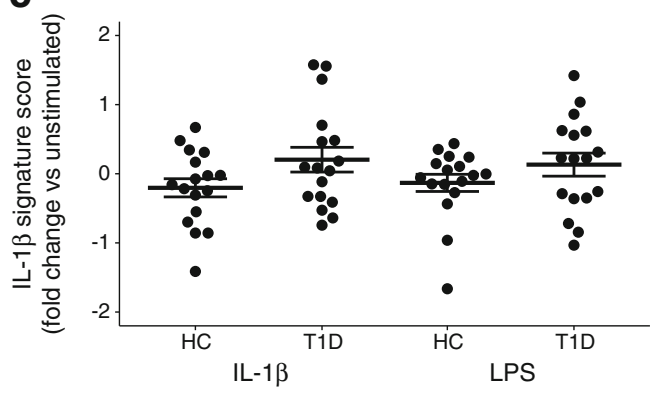

Fig. 5 IL-1-associated responses are similar in type 1 diabetes (T1D) and healthy control (HC) participants. (a, b) Volcano plots showing genes with significantly differential induction between T1D and HC samples after stimulation with IL-1 $\beta$ (a) or LPS (b). Horizontal dashed lines indicate adjusted $(\operatorname{adj}) p=0.05$; vertical dashed lines indicate $\log$ fold change equals 0 . (c) Milieu Intérieur-derived IL- $1 \beta$-specific gene signature scores for samples from T1D or HC participants after stimulation with IL- $1 \beta$ or LPS. For IL- $1 \beta, p=0.076$; for LPS, $p=0.21$ dependent gene expression, we found a significant increase in the IFN-1-driven gene signature in type 1 diabetes, as compared with healthy control participants, with both IFN- $\beta$ and polyIC stimulation (Fig. $6 \mathrm{c}$ ). A heatmap of the genes in the Milieu Intérieur-derived IFN- $\beta$ module after IFN- $\beta$ stimulation shows higher expression of IFN-1-induced genes in individuals with type 1 diabetes (Fig. 6d, ESM Fig. 4a). A heatmap showing the pattern of key signature scores in healthy control and type 1 diabetic individuals from this cohort is presented in ESM Fig. 4b. Enrichment of genes differentially induced in type 1 diabetes after IFN- $\beta$ or polyIC stimulation was then tested using the broader MSigDB hallmark set of IFN-1 response genes and a similar pattern was observed, with upregulation of a significant proportion of the IFN-response genes in individuals with type 1 diabetes, as compared with healthy control participants (Fig. $6 \mathrm{e})$.

Differential cellular composition could contribute to the cytokine levels or gene expression changes observed in whole blood. We compared major cell populations from cell blood counts (CBCs) between type 1 diabetes and healthy control participants. In our cohort, monocyte levels were higher in type 1 diabetes than in the healthy control group (ESM Fig. $5 \mathrm{a})$; however, levels of monocytes did not significantly correlate with IFN responses $\left(r^{2}=0.025, p=0.39\right)$, and the differences in IFN response gene expression were not explained by cell levels (ESM Fig. 5b, c). Further, neither demographic nor clinical variables were associated with stimulation-induced IFN gene expression, either in individual genes (ESM Fig. 1) or gene signature scores (ESM Fig. 6).

Specific genes from the Milieu Intérieur-derived IFN- $\beta$ module may be relevant for type 1 diabetes pathogenesis. For example, IFIH1, an ISG linked to type 1 diabetes, shows higher expression after IFN- $\beta$, polyIC and LPS stimulation in samples from individuals with type 1 diabetes (Fig. 6a). $X C R 1$, which specifically marks the cross-presenting conventional type I dendritic cells (cDC1s) that are critical for inducing both cytotoxic CD8 T cells and Th1 responses, is upregulated after polyIC stimulation in type 1 diabetes vs healthy control participants (ESM Fig. 7). In addition, IL12RB1 is induced more in type 1 diabetes compared with the healthy control group with all three IFN-1-inducing stimuli (LPS, polyIC and IFN- $\beta$ ). As IL-12 signalling is critical for Th1 response induction, this may contribute to the Th1 bias described in type 1 diabetes [48, 52].

To assess whether the observed alterations in the IFN-1 response were due to differential induction of IFN-1 proteins, we measured IFN- $\alpha$ and IFN- $\beta$ protein levels after polyIC stimulation by digital ELISA [36]. No significant differences in cytokine protein levels were observed between the type 1 diabetes and healthy control groups (Fig. 6f, g). Therefore, the observed differences may instead be due to alterations in IFN1 signalling. Consistent with this hypothesis, several genes downstream of the IFNAR showed higher IFN- $\beta$-induced upregulation in type 1 diabetes, including JAK2, STAT1 and STAT2 (Fig. 6a and ESM Fig. 7). Together, these data show an altered IFN-1 response in individuals with type 1 diabetes, with a higher dynamic range than control participants due to both lower baseline expression and higher stimulated expression of IFN-1 genes.

\section{Discussion}

In this study, we show that measuring gene expression after whole blood ex vivo stimulation allows identification of significant alterations in innate immune responsiveness in type 1 diabetes that are not detected without stimulation. Increased IFN-1 signalling, observed after IFN- $\beta$, polyIC and LPS stimulation, was the response that was most strongly associated with type 1 diabetes. Although gene expression induced by type 1 and type 2 IFNs have considerable overlap, 
a

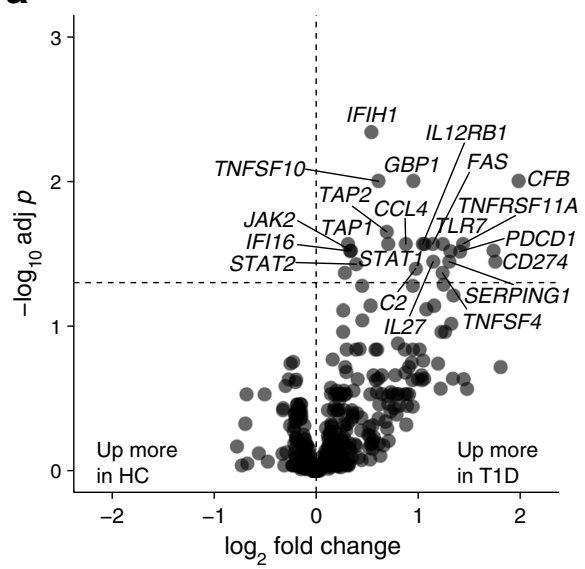

C

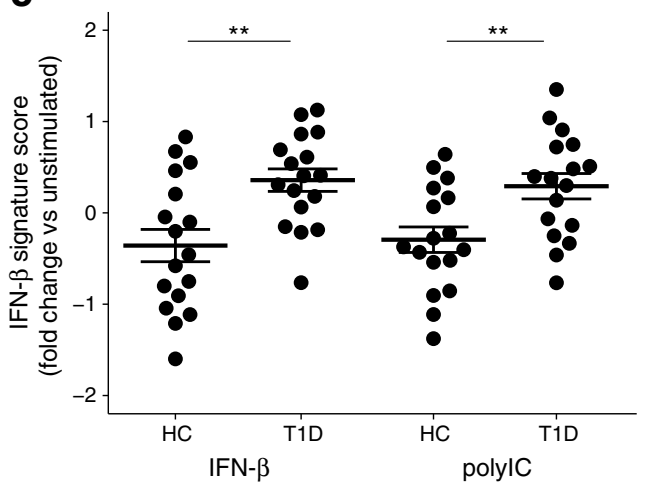

b

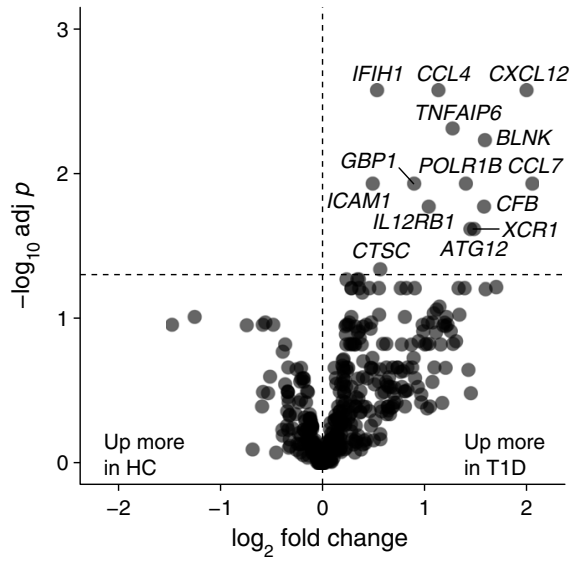

d

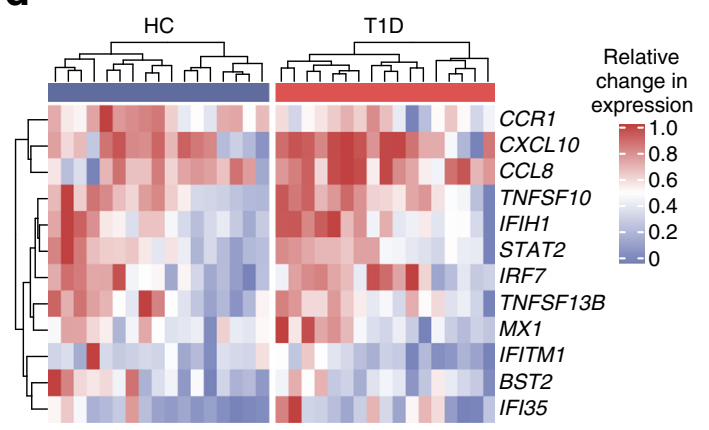

e

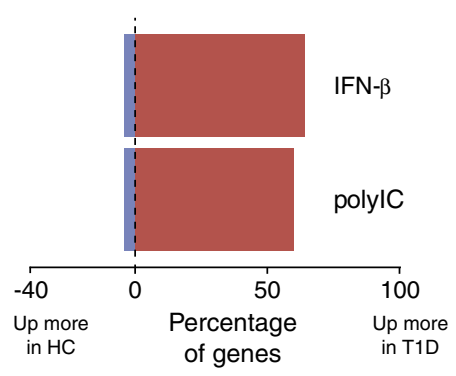

f

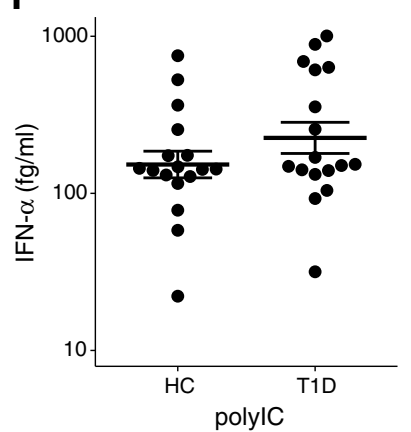

g

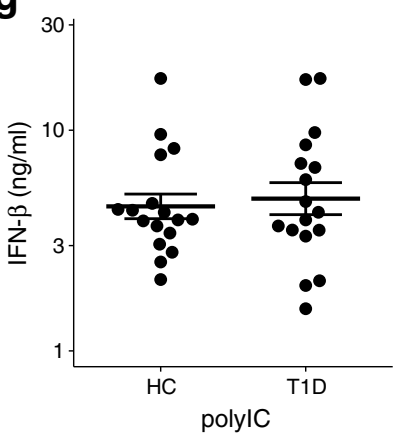

Fig. 6 Higher IFN-1-induced responses in type 1 diabetes (T1D) samples stimulated with IFN- $\beta$ or polyIC. $(\mathbf{a}, \mathbf{b})$ Volcano plots showing genes with significantly differential induction between T1D and healthy control (HC) samples after stimulation with IFN- $\beta$ (a) or polyIC (b). Horizontal dashed lines indicate adjusted $(\operatorname{adj}) p=0.05$; vertical dashed lines indicate $\log$ fold change equals 0 . (c) Milieu Intérieur-derived IFN- $\beta$-specific gene signature scores for samples from T1D or HC particpants after stimulation with IFN- $\beta$ and polyIC. For IFN- $\beta, p=0.0022$; for polyIC, $p=0.0056$. (d) Heatmap of IFN- $\beta$-induced expression of Milieu Intérieur-derived IFN- $\beta$-response genes in T1D and HC samples, with T1D and HC clustered separately. (e) Proportion of hallmark IFN- $\alpha$ response genes preferentially induced in T1D vs HC. (f, g) IFN- $\alpha$ (f) and IFN- $\beta$ (g) protein levels in supernatants from T1D or HC samples after polyIC stimulation, as measured by Quanterix Simoa assay. For IFN- $\alpha, p=0.20$; for IFN- $\beta, p=0.70$. Values shown on a $\log _{10}$ scale. $* * p<0.01$ vs HC the underlying pathways that lead to induction of both types of IFN and the downstream biological effects can differ greatly. Therefore, separating the two types of IFN responses is important for understanding the pathogenesis of autoimmune diabetes. The increased IFN-1 responsiveness, together with a lack of change in IFN- $\gamma$ signalling, suggests that individuals with type 1 diabetes are hyperresponsive to IFN-1, but not to type 2 IFN.

IL-1-induced responses after IL-1 $\beta$ or LPS stimulation were similar in individuals with established type 1 diabetes and healthy control participants. Although prior studies showed an IL-1-associated signature at the onset of type 1 
diabetes, our study did not show significant differences in IL1 -induced responses between type 1 diabetes and healthy control participants. This may be due to differences in experimental design or to unique phenotypic characteristics present in the peri-diagnostic period [53, 54]. It is also possible that there is, in fact, a difference in IL-1-induced responses, but that our study was not sufficiently powered to detect them. Indeed, some IL-1-induced differences were observed and, although not significant, the effect was in the expected direction. It is noteworthy, however, that blocking IL-1 signals in new-onset type 1 diabetes showed no clinical effect, suggesting that this pathway may not be essential for diabetes pathogenesis [29]. The adaptive immune responses tested here using SEB were not significantly different between groups, perhaps due to an absence of antigen-driven autoreactive responses. Nevertheless, these results do show that individuals with type 1 diabetes do not have a substantive difference in responsiveness to global adaptive immune cell activation as compared with control participants.

Some, but not all aspects of innate immune response alterations in human type 1 diabetes are reflected in autoimmune NOD mice. ISG expression is lower at baseline in monocytes from prediabetic NOD mice with chronic autoimmunity, but higher after acute IFN-inducing stimulation. Interestingly, this phenotype of reduced tonic IFN-1 signals was stronger in mouse monocytes and monocyte-derived dendritic cells (moDCs) as compared with conventional dendritic cells (cDCs) and plasmacytoid dendritic cells (pDCs), which are in low numbers in the blood [16]. Our earlier work focusing on gene expression in specific dendritic cell subsets after $\mathrm{CpG}$ stimulation did not show increased ISG expression, but these populations are rare in whole blood and may have differential autoimmune-driven changes in IFN-1 responses [55]. Future studies isolating specific cell populations may identify type 1 diabetes-associated alterations in human cDC IFN-1 responses. Although NOD mice may model type 1 diabetesassociated IFN responses, our data suggest that IL-1 responses may differ between mice and humans because prediabetic NOD mice with ongoing autoimmunity displayed higher baseline IL-1 responses [16]. Increased IL-1 may be transient, being present at the time of onset but fading in human type 1 diabetes over time [23, 56]. The human participants in our study had established type 1 diabetes, with time from diagnosis ranging from 2 to 8 years. Further work is needed to resolve this discrepancy.

The ability to observe significant alterations with a relatively small number of individuals illustrates the advantage of using whole blood ex vivo stimulation with gene signature analysis, but further data with an independent cohort would help strengthen the conclusions of this study. Although the study size limits conclusions regarding participant subsets, heterogeneity in ISG responses was observed within both the type 1 diabetes and healthy control group. Assessing gene expression after ex vivo stimulation in larger participant cohorts would allow the integration of genetic susceptibility with induced gene expression phenotypes and may potentially help to identify type 1 diabetes immune subtypes for differential therapeutic interventions. Including first-degree relatives of individuals with type 1 diabetes in future studies could further facilitate understanding of how genetic risk contributes to altered innate immune response. Identification of the cell types involved and the regulatory mechanisms contributing to the differential IFN-1 response in type 1 diabetes could also enable more precise therapeutic targeting of this pathway. Because of the more robust gene expression changes occurring with stimulation, its use in the context of current interventional trials could facilitate novel biomarker identification.

We propose a model incorporating what we now know about the complex nature of IFN-1 responses in type 1 diabetes. Early in disease pathogenesis (before onset or even autoantibody seroconversion), baseline IFN-1 response genes are increased $[18,19]$, likely due to low-level increases in IFN- $\alpha$ and IFN- $\beta$ expression. This chronic IFN exposure over time may suppress the tonic, unstimulated response, but prime a stronger acute response, possibly via epigenetic changes [57]. This could explain why unstimulated ISG expression is high in individuals at risk for type 1 diabetes $[19,26]$ and lower in established type 1 diabetes [58]. However, with strong acute stimulation, increased ISG expression is observed even after disease onset [59]. Additional work is needed to validate and better understand the changes in IFN response during the course of human type 1 diabetes. Using the TruCulture system at earlier disease stages, from before autoantibody seroconversion through to diabetes onset, could be informative for defining IL-1 and IFN-1 responses. Overall, this work shows the value in measuring both direct ex vivo and stimulated states for characterising how innate immunity is altered in autoimmune diseases such as type 1 diabetes.

Acknowledgments We thank J. Snavely and other members of the BRI Translational Research Program (Seattle, WA, USA) for their efforts in recruiting participants for this study. We also thank R. Hartley (also at the BRI) for her efforts in the initial processing of samples for this study, especially those at very early hours of the day. We acknowledge the UTechS CB platform of the Center for Translational Research, Institut Pasteur (Paris, France) for Nanostring data generation.

Data availability Mouse gene expression data are found in the gene expression omnibus (GEO) repository, accession GSE146452 (www. ncbi.nlm.nih.gov/geo/query/acc.cgi?acc=GSE146452). Nanostring count data from the human experiments were deposited in the GEO repository, accession GSE146338 (www.ncbi.nlm.nih.gov/geo/query/ acc.cgi?acc=GSE146338). Data files and $\mathrm{R}$ code for all analyses are available at https://github.com/rodriguesk/T1D_truculture_diabetologia.

Funding This work was supported by the intramural research programme of the National Institute of Diabetes and Digestive and Kidney Diseases (NIDDK)/National Institutes of Health (NIH). We thank Janssen Pharmaceuticals incorporated for financial support for the mouse gene expression data and Vipul Bhargava at Janssen for technical support in 
analysing the mouse gene expression data. Janssen was not involved otherwise in the design of the study; the collection, analysis, and interpretation of data; writing the report; and did not impose any restrictions regarding the publication of the report. DD acknowledges support from the Milieu Intérieur Consortium (10-LABX-69-01), from the Agence National de Recherche (ANR CE17001002) and Immunoqure for provision of monoclonal antibodies for the Quanterix Simoa IFN- $\alpha$ assay.

Authors' relationships and activities KVT is a shareholder and full-time employee of Amgen, Inc. The remaining authors declare that there are no relationships or activities that might bias, or be perceived to bias, their work.

Contribution statement KBR and MJD performed the bioinformatic data analysis and wrote the manuscript; MJR designed the mouse experiments; AL, MJR and VB conducted experiments and analysed data and edited the manuscript; CS helped design the human study, coordinated participant sample collection and edited the manuscript; JQ analysed data and contributed to drafting the article; PSL analysed data and edited the manuscript; CJG designed the human study and edited the manuscript; DD provided reagents, analysed data, designed the study and edited the manuscript; KVT designed and coordinated the study, analysed data and wrote the manuscript. DD and KVT are responsible for the integrity of the work as a whole. All authors approved of the final version of this manuscript.

\section{References}

1. Price JD, Tarbell KV (2015) The role of dendritic cell subsets and innate immunity in the pathogenesis of type 1 diabetes and other autoimmune diseases. Front Immunol 6:288. https://doi.org/10. 3389/fimmu.2015.00288

2. Liang Y, Sarkar MK, Tsoi LC, Gudjonsson JE (2017) Psoriasis: a mixed autoimmune and autoinflammatory disease. Curr Opin Immunol 49:1-8. https://doi.org/10.1016/j.coi.2017.07.007

3. Arts RJW, Joosten LAB, Netea MG (2018) The potential role of trained immunity in autoimmune and autoinflammatory disorders. Front Immunol 9:298. https://doi.org/10.3389/fimmu.2018.00298

4. Marshak-Rothstein A (2016) Autoimmunity: promoting and stabilizing innate immunity 'UNWUCHT'. Immunol Rev 269(1):7-10. https://doi.org/10.1111/imr.12387

5. Hotta-Iwamura C, Tarbell KV (2016) Type 1 diabetes genetic susceptibility and dendritic cell function: potential targets for treatment. J Leukoc Biol 100(1):65-80.

6. Mayer-Barber KD, Yan B (2017) Clash of the cytokine titans: counter-regulation of interleukin-1 and type I interferon-mediated inflammatory responses. Cell Mol Immunol 14(1):22-35. https:// doi.org/10.1038/cmi.2016.25

7. Ludigs K, Parfenov V, Pasquier RAD, Guarda G (2012) Type I IFN-mediated regulation of IL-1 production in inflammatory disorders. Cell Mol Life Sci 69(20):3395-3418. https://doi.org/10.1007/ s00018-012-0989-2

8. McNab F, Mayer-Barber K, Sher A, Wack A, O'Garra A (2015) Type I interferons in infectious disease. Nat Rev Immunol 15(2): 87-103. https://doi.org/10.1038/nri3787

9. Davidson S, Maini MK, Wack A (2015) Disease-promoting effects of type I interferons in viral, bacterial, and coinfections. J Interf Cytokine Res 35(4):252-264. https://doi.org/10.1089/jir.2014.0227

10. Mostafavi S, Yoshida H, Moodley D et al (2016) Parsing the interferon transcriptional network and its disease associations. Cell 164(3):564-578. https://doi.org/10.1016/j.cell.2015.12.032
11. Mayer-Barber KD, Andrade BB, Oland SD et al (2014) Hostdirected therapy of tuberculosis based on interleukin-1 and type I interferon crosstalk. Nature 511(7507):99-103. https://doi.org/10. 1038/nature13489

12. Hall JC, Rosen A (2010) Type I interferons: crucial participants in disease amplification in autoimmunity. Nat Rev Rheumatol 6(1): 40-49. https://doi.org/10.1038/nrrheum.2009.237

13. Yang C-A, Chiang B-L (2015) Inflammasomes and human autoimmunity: a comprehensive review. J Autoimmun 61:1-8. https:// doi.org/10.1016/j.jaut.2015.05.001

14. Diana J, Simoni Y, Furio L et al (2013) Crosstalk between neutrophils, B-1a cells and plasmacytoid dendritic cells initiates autoimmune diabetes. Nat Med 19(1):65-73. https://doi.org/10.1038/nm. 3042

15. Li Q, Xu B, Michie SA, Rubins KH, Schreriber RD, Mcdevitt HO (2008) Interferon-alpha initiates type 1 diabetes in nonobese diabetic mice. Proc Natl Acad Sci U S A 105(34):12439-12444. https:// doi.org/10.1073/pnas.0806439105

16. Rahman MJ, Rodrigues KB, Quiel JA et al (2018) Restoration of the type I IFN-IL-1 balance through targeted blockade of PTGER4 inhibits autoimmunity in NOD mice. JCI insight 3(3):e97843. https://doi.org/10.1172/jci.insight.97843

17. Rodriguez-Calvo T, Sabouri S, Anquetil F, von Herrath MG (2016) The viral paradigm in type 1 diabetes: who are the main suspects? Autoimmun Rev 15(10):964-969. https://doi.org/10.1016/j.autrev. 2016.07.019

18. Kallionpaa H, Elo LL, Laajala E et al (2014) Innate immune activity is detected prior to seroconversion in children with HLA-conferred type 1 diabetes susceptibility. Diabetes 63(7):2402-2414. https:// doi.org/10.2337/db13-1775

19. Ferreira RC, Guo H, Coulson RMR et al (2014) A type I interferon transcriptional signature precedes autoimmunity in children genetically at risk for type 1 diabetes. Diabetes 63(7):2538-2550. https:// doi.org/10.2337/db13-1777

20. Lundberg M, Krogvold L, Kuric E, Dahl-Jørgensen K, Skog O (2016) Expression of interferon-stimulated genes in insulitic pancreatic islets of patients recently diagnosed with type 1 diabetes. Diabetes 65(10):3104-3110. https://doi.org/10.2337/db16-0616

21. Marroqui L, Dos Santos RS, Op de beeck A et al (2017) Interferon$\alpha$ mediates human beta cell HLA class I overexpression, endoplasmic reticulum stress and apoptosis, three hallmarks of early human type 1 diabetes. Diabetologia 60(4):656-667. https://doi.org/10. 1007/s00125-016-4201-3

22. Foulis AK, Farquharson MA, Meager A (1987) Immunoreactive $\alpha$ interferon in insulin-secreting $\beta$ cells in type 1 diabetes mellitus. Lancet 330(8573):1423-1427. https://doi.org/10.1016/S01406736(87)91128-7

23. Hussain MJ, Peakman M, Gallati H et al (1996) Elevated serum levels of macrophage-derived cytokines precede and accompany the onset of IDDM. Diabetologia 39(1):60-69. https://doi.org/10. 1007/BF00400414

24. Bradshaw EM, Raddassi K, Elyaman W et al (2009) Monocytes from patients with type 1 diabetes spontaneously secrete proinflammatory cytokines inducing Th17 cells. J Immunol 183(7):44324439. https://doi.org/10.4049/jimmunol.0900576

25. Cabrera SM, Wang X, Chen Y-G et al (2016) Interleukin-1 antagonism moderates the inflammatory state associated with type 1 diabetes during clinical trials conducted at disease onset. Eur J Immunol 46(4):1030-1046. https://doi.org/10.1002/eji.201546005

26. Chen Y-G, Cabrera SM, Jia S et al (2014) Molecular signatures differentiate immune states in type 1 diabetic families. Diabetes 63(11):3960-3973. https://doi.org/10.2337/db14-0214

27. Larsen CM, Faulenbach M, Vaag A et al (2007) Interleukin-1receptor antagonist in type 2 diabetes mellitus. $\mathrm{N}$ Engl $\mathrm{J}$ Med 356(15):1517-1526. https://doi.org/10.1056/NEJMoa065213 
28. Nikfar S, Saiyarsarai P, Tigabu BM, Abdollahi M (2018) Efficacy and safety of interleukin-1 antagonists in rheumatoid arthritis: a systematic review and meta-analysis. Rheumatol Int 38(8):13631383. https://doi.org/10.1007/s00296-018-4041-1

29. Moran A, Bundy B, Becker DJ et al (2013) Interleukin-1 antagonism in type 1 diabetes of recent onset: two multicentre, randomised, double-blind, placebo-controlled trials. Lancet 381(9881):1905-1915. https://doi.org/10.1016/S0140-6736(13) 60023-9

30. Kayserova J, Vcelakova J, Stechova K et al (2014) Decreased dendritic cell numbers but increased TLR9-mediated interferonalpha production in first degree relatives of type 1 diabetes patients. Clin Immunol 153(1):49-55. https://doi.org/10.1016/j.clim.2014. 03.018

31. Duffy D, Rouilly V, Libri V et al (2014) Functional analysis via standardized whole-blood stimulation systems defines the boundaries of a healthy immune response to complex stimuli. Immunity 40(3):436-450. https://doi.org/10.1016/j.immuni.2014.03.002

32. Urrutia A, Duffy D, Rouilly V et al (2016) Standardized wholeblood transcriptional profiling enables the deconvolution of complex induced immune responses. Cell Rep 16(10):2777-2791. https://doi.org/10.1016/j.celrep.2016.08.011

33. Duffy D, Rouilly V, Braudeau C et al (2017) Standardized whole blood stimulation improves immunomonitoring of induced immune responses in multi-center study. Clin Immunol 183:325-335. https://doi.org/10.1016/j.clim.2017.09.019

34. Guerrero AD, Dong MB, Zhao Y, Lau-Kilby A, Tarbell KV (2014) Interleukin-2-mediated inhibition of dendritic cell development correlates with decreased CD135 expression and increased monocyte/macrophage precursors. Immunology 143(4):640-650. https://doi.org/10.1111/imm. 12345

35. Dong MB, Rahman MJ, Tarbell KV (2016) Flow cytometric gating for spleen monocyte and DC subsets: differences in autoimmune NOD mice and with acute inflammation. J Immunol Methods 432: 4-12. https://doi.org/10.1016/j.jim.2015.08.015

36. Rodero MP, Decalf J, Bondet V et al (2017) Detection of interferon alpha protein reveals differential levels and cellular sources in disease. J Exp Med 214(5):1547-1555. https://doi.org/10.1084/ jem.20161451

37. Llibre A, Bilek N, Bondet V et al (2019) Plasma type I IFN protein concentrations in human tuberculosis. Front Cell Infect Microbiol 9:296. https://doi.org/10.3389/fcimb.2019.00296

38. Vandesompele J, De Preter K, Pattyn F et al (2002) Accurate normalization of real-time quantitative RT-PCR data by geometric averaging of multiple internal control genes. Genome Biol 3(7): research0034. https://doi.org/10.1186/gb-2002-3-7-research0034

39. Armbruster DA, Pry T (2008) Limit of blank, limit of detection and limit of quantitation. Clin Biochem Rev 29(Suppl 1):S49-S52

40. Johnson WE, Li C, Rabinovic A (2007) Adjusting batch effects in microarray expression data using empirical Bayes methods. Biostatistics 8(1):118-127. https://doi.org/10.1093/biostatistics/ kxj037

41. Leek JT, Johnson WE, Parker HS, Jaffe AE, Storey JD (2012) The sva package for removing batch effects and other unwanted variation in high-throughput experiments. Bioinformatics 28(6):882883. https://doi.org/10.1093/bioinformatics/bts034

42. Ritchie ME, Phipson B, Wu D et al (2015) Limma powers differential expression analyses for RNA-sequencing and microarray studies. Nucleic Acids Res 43(7):e47. https://doi.org/10.1093/nar/ gkv007

43. Gu Z, Eils R, Schlesner M (2016) Complex heatmaps reveal patterns and correlations in multidimensional genomic data. Bioinformatics 32(18):2847-2849. https://doi.org/10.1093/ bioinformatics/btw313

44. Wu D, Lim E, Vaillant F, Asselin-Labat M-L, Visvader JE, Smyth GK (2010) ROAST: rotation gene set tests for complex microarray experiments. Bioinformatics 26(17):2176-2182. https://doi.org/10. 1093/bioinformatics/btq401

45. Liberzon A, Birger C, Thorvaldsdóttir H, Ghandi M, Mesirov JP, Tamayo P (2015) The Molecular Signatures Database (MSigDB) hallmark gene set collection. Cell Syst 1(6):417-425. https://doi. org/10.1016/J.CELS.2015.12.004

46. R Core Team (2018) R: a language and environment for statistical computing. Available from: https://www.R-project.org/. Accessed: 20 April 2019

47. Wickham H (2016) ggplot2: elegant graphics for data analysis, 2nd edn. Springer-Verlag, New York, NY. https://doi.org/10.1007/9783-319-24277-4

48. Arif S, Tree TI, Astill TP et al (2004) Autoreactive T cell responses show proinflammatory polarization in diabetes but a regulatory phenotype in health. J Clin Invest 113(3):451-463. https://doi.org/ 10.1172/JCI19585

49. Haskins K, Cooke A (2011) CD4 T cells and their antigens in the pathogenesis of autoimmune diabetes. Curr Opin Immunol 23(6): 739-745. https://doi.org/10.1016/J.COI.2011.08.004

50. Cabrera SM, Henschel AM, Hessner MJ (2016) Innate inflammation in type 1 diabetes. Transl Res 167(1):214-227. https://doi.org/ 10.1016/j.trsl.2015.04.011

51. Timmerman JJ, Verweij CL, van Gijlswijk-Janssen DJ, van der Woude FJ, van Es LA, Daha MR (1995) Cytokine-regulated production of the major histocompatibility complex class-IIIencoded complement proteins factor $\mathrm{B}$ and $\mathrm{C} 4$ by human glomerular mesangial cells. Hum Immunol 43(1):19-28. https://doi.org/ 10.1016/0198-8859(94)00122-7

52. Walker LSK, von Herrath M (2016) CD4 T cell differentiation in type 1 diabetes. Clin Exp Immunol 183(1):16-29. https://doi.org/ 10.1111/cei.12672

53. Fatima N, Faisal SM, Zubair S et al (2016) Role of proinflammatory cytokines and biochemical markers in the pathogenesis of type 1 diabetes: correlation with age and glycemic condition in diabetic human subjects. PLoS One 11(8):e0161548. https://doi. org/10.1371/journal.pone.0161548

54. Akbari M, Hassan-Zadeh V (2018) Hyperglycemia affects the expression of inflammatory genes in peripheral blood mononuclear cells of patients with type 2 diabetes. Immunol Investig 47(7):654 665. https://doi.org/10.1080/08820139.2018.1480031

55. Rahman MJ, Rahir G, Dong MB et al (2016) Despite increased type 1 IFN, autoimmune nonobese diabetic mice display impaired dendritic cell response to $\mathrm{CpG}$ and decreased nuclear localization of IFN-activated STAT1. J Immunol 196(5):2031-2040. https:// doi.org/10.4049/jimmunol.1501239

56. Levy H, Wang X, Kaldunski M et al (2012) Transcriptional signatures as a disease-specific and predictive inflammatory biomarker for type 1 diabetes. Genes Immun 13(8):593-604. https://doi.org/ 10.1038/gene.2012.41

57. Park SH, Kang K, Giannopoulou E et al (2017) Type I interferons and the cytokine TNF cooperatively reprogram the macrophage epigenome to promote inflammatory activation. Nat Immunol 18(10):1104-1116. https://doi.org/10.1038/ni.3818

58. Kreuzer D, Nikoopour E, Au BCY et al (2015) Reduced interferon$\alpha$ production by dendritic cells in type 1 diabetes does not impair immunity to influenza virus. Clin Exp Immunol 179(2):245-255. https://doi.org/10.1111/cei.12462

59. Xia CQ, Peng R, Chernatynskaya AV et al (2014) Increased IFN- $\alpha$ producing plasmacytoid dendritic cells (pDCs) in human Th1mediated type 1 diabetes: pDCs augment Th1 responses through IFN- $\alpha$ production. J Immunol 193(3):1024-1034. https://doi.org/ 10.4049/jimmunol.1303230

Publisher's note Springer Nature remains neutral with regard to jurisdictional claims in published maps and institutional affiliations. 